\title{
Measurement of Ki-67 antigen in 159 pituitary adenomas using the MIB-1 monoclonal antibody
}

\section{C.B. Pizarro ${ }^{1}$, M.C. Oliveira ${ }^{1}$, L.B. Coutinho ${ }^{2}$ and N.P. Ferreira ${ }^{3}$}

Departamentos de ${ }^{1}$ Endocrinologia, ${ }^{2}$ Neuropatologia and ${ }^{3}$ Neurocirurgia, Fundação Faculdade Federal de Ciências Médicas de Porto Alegre, Porto Alegre, RS, Brasil
Correspondence

M.C. Oliveira

Rua Dona Mimi Moro, 40

90480-050 Porto Alegre, RS

Brasil

E-mail: mco@portoweb.com.br ................

Received October 28, 2002 Accepted September 23, 2003

\begin{abstract}
Pituitary adenomas sometimes show rapid growth and recurrence, and about one third invade the structures surrounding the sella turcica. In an attempt to determine aggressive behavior at an early stage, we used the MIB-1 antibody to identify the Ki-67 antigen. The present study was designed to evaluate pituitary adenomatous tissue in terms of secretion and proliferation and to correlate the Ki-67 index with hormone phenotype and invasive behavior. Material from 159 patients submitted to one or more resections of pituitary adenomas was evaluated. Forty-two non-secretory adenomas and 43 adenomas immunoreactive for growth hormone, 19 for prolactin, 18 for growth hormone and prolactin, 16 for adrenocorticotropic hormone (ACTH), and 21 cases of plurihormonal/gonadotropin adenomas were detected by immunohistochemistry. The MIB-1 antibody was positive in 139 samples and the Ki-67 index ranged from 0.16 to $15.48 \%$ (mean $=1.22$ $\pm 2.09 \%$ ), with no significant difference between genders, age groups, or secretory and non-secretory status. The Ki-67 index was higher in ACTH-secreting adenomas. Invasive pituitary adenomas had a significantly higher Ki-67 index $(2.01 \pm 3.15 \%)$ than macroadenomas with or without supra-sellar extension $(1.12 \pm 1.87 \%$; $=0.02)$. The index was not significantly different in the subgroup of adenomas with invasion of the cavernous sinus compared to groups with other types of invasion. We conclude that tumoral proliferative activity evaluated by the detection of the Ki-67 antigen is significantly higher in invasive than noninvasive adenomas, information which can be useful in therapeutic postoperative management since index cut-off values associated with aggressive behavior can be established.
\end{abstract}

Key words

- Pituitary adenoma

- Invasive adenoma

- Ki-67 antigen

- MIB-1 antibody

- Cell proliferation

- Immunohistochemistry

\section{Introduction}

Pituitary adenomas are benign neoplasias usually of slow growth. They are discovered incidentally at a frequency of about $20 \%$ in image scans of the area (1), in about $27 \%$ of the glands examined at autopsy (2), or by clinical evidence resulting from hormone hypo- or hypersecretion or symptoms of intracranial mass. When they produce hormones in excess, they may have severe metabolic consequences which lead to significant morbidity and mortality (3). One third of all pituitary adenomas invade the structures sur- 
rounding the sella turcica, with rapid growth and/or postoperative recurrence. The incidence of invasion by pituitary tumors varies according to the method of diagnosis used histological, surgical or radiological - ranging from 10 to $85 \%$, and is higher when determined on the basis of microscopic invasion of the dura mater. A number of techniques to evaluate cell proliferation have been used in order to identify adenomas with potentially invasive characteristics at an early stage. Immunohistochemistry has been used to assess the aggressiveness of tumoral pituitary tissue by measuring cell proliferation indexes based on the detection of the Ki-67 antigen (4), which is present in all stages of the cell cycle but not in resting cells (5).

The objective of the present study was to detect the Ki-67 antigen using the MIB-1 antibody and to quantify it in a large sample of pituitary adenomas resected in our community, and to establish the relationship of the $\mathrm{Ki}-67$ index with hormone production assessed by immunohistochemistry and with the presence of tumor invasion.

\section{Subjects and Methods}

A total of 208 surgeries for resection of pituitary tumors were performed at a major neurosurgery hospital in South Brazil from January 1987 to December 1997. The medical records of these patients were reviewed for clinical presentation, hormone evaluation, pituitary image, and drug use. In cases of reintervention, the same data were collected and updated to the time of reintervention.

Patients were initially grouped according to the presence of the different clinical syndromes of pituitary hormone hypersecretion (acromegaly, Cushing's disease, amenorrheagalactorrhea syndrome) or to the lack of clinical evidence for hormone hypersecretion. One hundred and twenty-one patients underwent a single surgery, 36 underwent two surgeries, and 2 underwent three surgeries.
Since a few paraffin blocks of pituitary tissue could not be located and since in some cases insufficient material was available and the medical records of the patients were not available, the final sample was reduced to 180 paraffin blocks from 159 patients (69 males and 90 females).

The study was approved by the University School Ethics Committee.

\section{Histology}

Paraffin blocks were cut into $3-\mu \mathrm{m}$ sections and mounted on glass slides prepared with organosilane.

For hormone phenotype determination, the primary polyclonal antibody developed in rabbits was used at the following dilutions: 1:7000 for growth hormone $(\mathrm{GH})$, 1:2000 for adrenocorticotropic hormone (ACTH), 1:1000 for luteinizing hormone, 1:3000 for follicle-stimulating hormone, 1:2000 for thyroid-stimulating hormone, and 1:1000 for prolactin (PRL) (Dakopatts, Santa Barbara, CA, USA). For the detection of the Ki-67 antigen, the MIB-1 antibody developed in rats was used at a dilution of 1:20 (Immunotech, Marseille, France).

All immunohistochemical procedures were performed using the avidin-biotin technique.

Slides were prepared simultaneously using the same antibody. Normal pituitary tissue obtained from autopsies was used for positive controls for hormone detection, and neoplastic breast tissue was used for positive controls for MIB-1. The primary antibody was replaced with normal horse serum in negative controls.

The sections submitted to the immunohistochemical technique were examined under a standard light microscope. Proliferative activity, assessed by the MIB-1 antibody, was measured in areas of high density of labeled cells. A reticulate slide attached to the microscope was used at a magnification of 400X. Cells labeled for the antibody were 
counted and their fraction of the total number of cells was determined, with the resulting value being reported as percent. In 110 cases $(84 \%)$ the count was performed in 4 to 6 fields (mean \pm SD of total cells counted $=$ $2147.9 \pm 877.5)$. In the other 29 cases (16\%), because of the quantity of material obtained, the mean \pm SD number of total cells counted was $840.7 \pm 457.7$.

The slides were read for hormone phenotype and MIB-1 antibody by the same person, who was not aware of the patient's clinical history.

\section{Tumor classification}

Tumors were classified as microadenomas $(<10 \mathrm{~mm})$ or macroadenomas $(\geq 10 \mathrm{~mm})$ according to size, with or without suprasellar extension. Imaging techniques (computed tomography or magnetic resonance) were used to define tumor invasion. Suprasellar growth was considered to be an extension rather than an invasion. Invasive adenomas were those which showed invasion of the cavernous, sphenoid or ethmoidal sinus, bone, and third ventricle, or with subfrontal growth.

\section{Statistical analysis}

The Student $t$-test and ANOVA followed by the Duncan test were used to compare two groups and more than two groups, respectively, with the level of significance set at $\mathrm{P}<0.05$.

\section{Results}

\section{Clinical results}

The sample consisted of 90 females ranging in age from 16 to 76 years (mean $\pm \mathrm{SD}=$ $43 \pm 14.5$ years) and 69 males ranging in age from 13 to 68 years ( $45.6 \pm 12.3$ years).

The group of patients with clinical evidence of acromegaly consisted of 48 patients
(30.2\% of the total sample) who were subdivided into two subgroups: 44 patients, 29 females and 15 males, whose clinical presentation was predominantly of acromegaly, and four patients whose acromegalic condition was associated with the amenorrheagalactorrhea syndrome or impotence. In the first subgroup, 9 patients showed clinical acromegaly in association with symptoms of the presence of an intracranial mass. Mean age was 46 years for females and 38.8 years for males. In the second subgroup one patient was a 61-year-old female and the other 3 were males aged 35,44 , and 52 years; all of them showed symptoms of the presence of an intracranial mass.

In the group with clinical evidence of Cushing's disease $(\mathrm{N}=22)$ or Nelson's syndrome $(\mathrm{N}=1)$, comprising $14.4 \%$ of the sample, 5 patients showed symptoms of the presence of an intracranial mass. Seventeen were females (mean age $=36.5$ years) and 6 were males (mean age $=34.5$ years).

The group of patients with clinical evidence of prolactinoma (amenorrhea-galactorrhea syndrome or impotence) comprised 33 cases (20.7\% of the sample), 27 of whom showed symptoms of the presence of an intracranial mass. Eighteen were females and 15 were males. Mean age was 36.3 years for females and 46.8 years for males.

Fifty-five patients ( $34.5 \%$ of the sample) did not show clinical evidence of endocrine dysfunction. Three tumors appeared to be incidentalomas and the others were detected as a result of symptoms of the presence of an intracranial mass. Twenty-five patients were females, with a mean age of 49 years, and 30 were males, with a mean age of 50.9 years.

\section{Sellar image evaluation}

Computed tomography and/or magnetic resonance of the sellar region were available in 135 medical records ( $85 \%$ of the sample). No lesion of the sella turcica was detected in 6 cases ( 5 females and one male). Micro- 
adenomas were detected in 19 cases (12 females and 7 males) and macroadenomas in 110 cases ( 60 females and 50 males). Among the macroadenomas, 42 showed isolated suprasellar extension, and the other 42 were considered to be invasive ( 7 with invasion of the cavernous sinus, 6 with invasion of the sphenoid sinus, 4 with parasellar growth, 13 with subfrontal growth, 4 with invasion of the third ventricle [one of them with hydrocephalus], 2 with invasion of the cavernous sinus and sphenoid sinus, 3 with subfrontal growth and growth toward the sphenoid sinus, one with invasion of the sphenoid, ethmoidal, and cavernous sinuses, and 2 with suprasellar growth and hydrocephalus). Therefore, $31.1 \%$ of the adenomas showed some degree of invasion. The presence of intratumoral calcifications detected by imaging was reported in 3 cases. Table 1 shows the sellar imaging findings for the different clinical presentations.

\section{Hormones detected by immunohistochemistry}

Forty-two adenomas (26.4\% of the sample) did not show immunoreaction to any of the adenohypophyseal hormones. Of 117 secretory cases $(73.5 \%), 79$ were immunoreactive to a single hormone $(67.5 \%)$, and the remaining ones were immunoreactive to two or more hormones. The frequency of hor-

Table 1. Sellar imaging data for patients with pituitary adenomas.

\begin{tabular}{|c|c|c|c|c|c|}
\hline Clinical presentation & $\begin{array}{l}\text { Normal } \\
\text { image }\end{array}$ & $\begin{array}{c}\text { Micro- } \\
\text { adenoma }\end{array}$ & $\begin{array}{l}\text { Macro- } \\
\text { adenoma } \\
\text { restricted } \\
\text { to sella }\end{array}$ & $\begin{array}{c}\text { Macro- } \\
\text { adenoma } \\
\text { with supra- } \\
\text { sellar extension }\end{array}$ & $\begin{array}{c}\text { Macro- } \\
\text { adenoma } \\
\text { with } \\
\text { invasion }\end{array}$ \\
\hline Acromegaly & 2 & 9 & 12 & 12 & 4 \\
\hline $\begin{array}{l}\text { Cushing's disease } \\
\text { or Nelson's syndrome }\end{array}$ & 4 & 9 & 2 & 2 & - \\
\hline $\begin{array}{l}\text { Amenorrhea-galactorrhea } \\
\text { syndrome or impotence }\end{array}$ & - & 1 & 6 & 6 & 17 \\
\hline Nonfunctioning adenoma & - & - & 6 & 22 & 21 \\
\hline Total & 6 & 19 & 26 & 42 & 42 \\
\hline
\end{tabular}

mone phenotypes was GH in 43 cases, PRL in 19 cases, ACTH in 16 cases, association of GH and PRL in 18 cases, and other associations in 21 cases.

Of the 42 adenomas which had invasive characteristics by tomography, 13 were negative for the pituitary hormones analyzed, 9 were immunoreactive for $\mathrm{GH}$ and PRL, 8 for PRL, 4 for GH, 3 for ACTH, and 5 for other hormonal associations. The most prevalent hormones in the invasive adenomas were PRL (17 cases) and GH (14 cases).

It was possible to examine the material from 23 of the patients submitted to subsequent pituitary intervention $(\mathrm{N}=32)$. Twelve of them did not show a positive reaction for any of the hormones analyzed, and again the most prevalent hormones produced were $\mathrm{GH}$ (10 cases) and PRL ( 9 cases).

\section{MIB-1 antibody}

The MIB-1 antibody was applied to 180 samples and was positive in 139 . The mean \pm SD Ki-67 index was $1.22 \pm 2.09 \%$ for the total sample (range: $0.16-15.48 \%$ ). The Ki67 index did not show statistically significant gender differences: $1.17 \pm 2.27 \%$ for males and $1.25 \pm 1.94 \%$ for females. For statistical analysis, patient age was divided into quartiles: up to 34 years ( $\mathrm{N}=42$ ), from 35 to 44 years $(\mathrm{N}=44)$, from 45 to 54 years $(\mathrm{N}=34)$, and over 55 years $(\mathrm{N}=39)$. The Ki67 index was not significantly different across these age groups, with respective values of $1.77 \pm 2.96,1.25 \pm 2.19,0.75 \pm 0.65$, and $0.99 \pm 1.52 \%$.

In 15 cases the Ki-67 index was above $3 \%$. Of these, 14 patients showed macroadenomas, four with suprasellar growth and 10 with invasive behavior. Of the invasive macroadenomas, two showed invasion of the cavernous sinus, two of the sphenoid sinus, two showed subfrontal growth, two showed subfrontal growth and invasion of the sphenoid sinus, one showed invasion of the cavernous sphenoid sinus, and one para- 
sellar growth. Among the 15 cases, two adenomas showed a Ki-67 index above 10\%: in one of them, a non-secretory tumor with invasion of the sphenoid sinus, the index was $15.48 \%$, and in the other, an ACTHsecreting tumor with suprasellar growth, the index was $12.71 \%$.

The MIB-1 values for each hormone phenotype are reported in Figure 1. Although the mean Ki-67 index for ACTH-secreting adenomas tended to be higher than in the other ones, no statistically significant difference was found between the different patterns of hormone secretion ( $\mathrm{P}=0.87)$.

The Ki-67 index was $1.18 \pm 1.85 \%$ for secretory tumors and $1.31 \pm 2.64 \%$ for nonsecretory tumors, with no significant difference between these groups $(\mathrm{P}=0.71)$.

Figure 2 shows the values of the Ki-67 index according to tumor size and invasive behavior. For statistical analysis, cases with a normal sellar image were grouped with cases of microadenomas.

The Ki-67 index was significantly higher in adenomas with invasion than in macroadenomas with no invasion. Ten patients, one with a non-secreting adenoma and 9 with secretory adenomas, showed invasion of the cavernous sinus. Of the secretory adenomas, 3 were reactive for $\mathrm{GH}, 2$ for PRL, 2 for $\mathrm{GH}$ and PRL, and 2 for ACTH. No significant difference in the Ki-67 index was found between the group with invasion of the cavernous sinus $(2.34 \pm 2.49 \%)$ and the group with other types of invasion $(1.89 \pm 3.40 \%)$.

Also, no significant difference in the Ki67 index was found between tissue from primary tumors $(1.25 \pm 2.13 \%)$ and tissue from subsequent surgical pituitary intervention $(0.87 \pm 1.39 \% ; \mathrm{P}=0.29)$.

\section{Discussion}

In the cases studied here, there was a predominance of incidence in the fifth decade of life. There was a slight predominance of pituitary adenomas in females over males (male:female ratio: $1: 1.13$ ). These data are in agreement with a previous study published in our community (6). Mindermann and Wilson (7) analyzed the gender incidence of different types of pituitary tumors according to clinical phenotype and found that prolactinomas and ACTH- and TSHsecreting adenomas occurred predominantly in females, while hormonally inactive adenomas and $\mathrm{GH}$-secreting adenomas occurred mainly in males. In the present study,

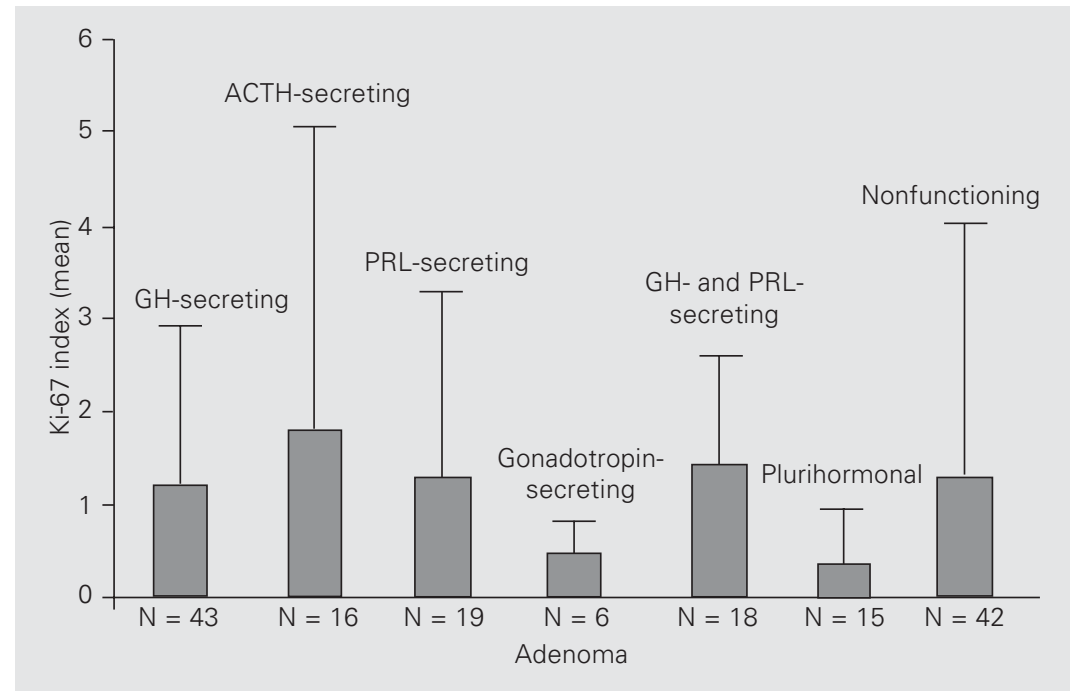

Figure 1. Ki-67 index and different patterns of hormone secretion. The Ki-67 index was determined with MIB-1 monoclonal antibodies and represents percent labeled cells. ACTH = adrenocorticotropic hormone; $\mathrm{GH}=$ growth hormone; $\mathrm{PRL}=$ prolactin .

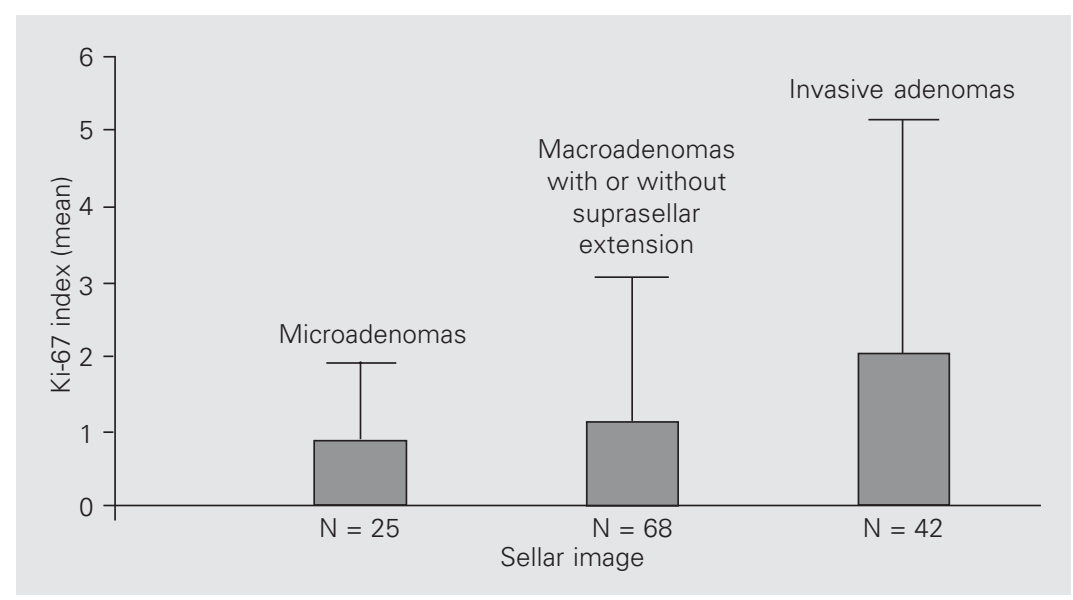

Figure 2. Ki-67 index and volume and extension of pituitary adenomas. The Ki-67 index was determined as described in the legend to Figure 1. The number of microadenomas includes 6 cases that were normal according to image analysis, but were positive according to tissue analysis. 
clinically GH-, ACTH- and PRL-secreting adenomas were predominant in females (1:1.6; $1: 2.8$ and $1: 1.2$, respectively), and nonfunctioning adenomas were predominant in males $(1.2: 1)$.

It is known that pituitary adenomas with aggressive biological behavior, such as invasion of underlying structures and rapid growth, have the clinical aspect of intermediate adenomas, i.e., with characteristics between those of adenomas and pituitary carcinomas. Histological alterations such as pleomorphism and aneuploidy are often observed, as well as alterations in cell proliferation markers. Tumor invasion leads to neurological complications and also impairs complete tumor excision and normalization of hormone levels. Tumor remnants usually continue to grow, requiring a new surgical intervention. The incidence of invasion by pituitary tumors is wide, ranging from $10 \%$ to $85 \%$ depending on the criterion used to define an adenoma as invasive or not, and is much higher when analyzed in terms of microscopic invasion of the dura mater. Using the criteria of gross invasion visualized by the surgeon and invasion of dura mater evaluated microscopically, Scheithauer et al. (8) found a frequency of $35 \%$ for invasion by adenomas. Invasion of $41.9 \%(24.1 \%$ in ACTH-secreting adenomas and $66.7 \%$ in tumors of null cells) was reported by other authors (9) also using the criterion of histological invasion of the dura mater. In the present study, an overall invasion rate of $31.1 \%$ was found, which was close to the one reported by Scheithauer et al. (8). This number could be greater, since the criterion for invasion used here was radiological (less sensitive), in contrast to the histological criteria used by other investigators. In our study, the highest percentage of invasion was detected for non-secreting (31\%) and GH- and PRL-secreting adenomas (21.4\%).

Invasion of the cavernous sinus occurs in $6-10 \%$ of all pituitary adenomas (10). Kawamoto et al. (11) reported that adenomas with invasion of the cavernous sinus had a significantly greater volume than those without invasion, although they showed no significant difference in the cell replication indexes assessed. The same investigators speculated that invasion of the cavernous sinus and growth rate are independent biological factors and that invasion of the cavernous sinus may be caused by chemical factors produced by the tumor itself. In the series described by Mastronardi et al. (12), 16 cases (15.5\%) showed invasion of the cavernous sinus diagnosed radiologically, with a significantly higher proliferative activity than observed in the group without invasion. In the present study, invasion of the cavernous sinus occurred in $7.4 \%$ of cases, in agreement with literature values.

The Ki-67 antigen reflects the state of the tumor cell at that moment of proliferation rather than the rate of cell proliferation. The antigen has been shown to be strongly correlated with thymidine labeling and flow cytometric determinations of S-phase fractions (13). The first reports of the Ki-67 index assessed specifically in pituitary adenomas concerned frozen or fresh material (14-19) while material embedded in paraffin was used later (20-22).

With respect to the identification of the antigen, Key et al. (23) reported three antibodies of recombinant parts of the Ki-67 antigen (MIB 1-3) and characterized them as true equivalents of Ki-67. Other studies confirmed the validity of comparative results with all antibodies cited (24). Since 1995, studies evaluating the MIB-1 monoclonal antibody in pituitary tumors have been reported in the literature $(11,12,25-33)$. In the studies by Knosp et al. (15), Zhao et al. (33) and Mastronardi et al. (12), the mean positive reaction to $\mathrm{Ki}-67$ was $1.1,1.4$, and $2.64 \%$, respectively. In the present study, the mean reaction to MIB-1 was $1.22 \%$, which is close to the values reported elsewhere.

In the examination of both fresh tissue and paraffin-embedded material, negative 
cases for Ki-67 antigen are found, as well as cases with percentages that markedly deviate from the mean. Using the MIB-1 antibody in paraffin-embedded sections, values of 3.6 through $16.4 \%$ have been reported. In the present study, the Ki-67 index was above $3 \%$ in 15 cases, and in two of them it was above $10 \%$, i.e., 12.71 and $15.48 \%$. Other investigators have reported values of $16.45 \%$ (28) and even $23.37 \%$ (11) in their series, values which are usually found in pituitary carcinomas.

With respect to index behavior according to patient gender and age, Yonezawa et al. (34) reported a significantly higher MIB-1 in nonfunctioning adenomas of patients younger than 30 years than in patients older than 40 years. Similarly, Jaffrain-Rea et al. (35) reported a significantly higher index in patients younger than 30 than in older patients. Another study, however, compared different age groups $(<25$, between 25 and $50,>50$ years) and found no significant difference in MIB-1 (12). The same finding was reported by others $(4,14)$, who did not detect any significant differences in Ki-67 index regarding patient gender or tumor diameter. Evaluating the Ki-67 index in prolactinomas, Delgrange et al. (30) found that the indexes were likely to be higher in males than in females, though such difference was not statistically significant. In the present study, there was no significant difference in the Ki-67 index among the different age groups analyzed. These results reflect the findings for the different age quartiles; however, individuals younger than 44 years tend to show higher values.

As regards the ratio between MIB-1 and clinical and/or hormone phenotype evaluated by immunohistochemistry, there is no agreement in published studies. While some investigators did not detect any difference in Ki-67 between active and inactive tumors $(11,15,18)$, others reported higher values for active tumors (28). Landolt et al. (14) examined the proliferative activity of 31 pituitary adenomas and reported higher Ki-67 values for acromegalic patients. Other studies reported a higher Ki-67 index for ACTH-secreting adenomas $(4,12)$ or PRL-secreting adenomas (25). In the present series, no significant difference was found between secretory and non-secretory adenomas or across the different groups of secretory adenomas, even though MIB-1 was higher in $\mathrm{ACTH}$-secreting adenomas and the second highest index in the series belonged to this phenotype.

Since the pioneering report by Landolt et al. (14), most studies assessing the Ki-67 index in pituitary adenomas have observed higher values in invasive adenomas, regardless of the criterion used to define invasion. Knosp et al. (15) reported a series of 62 pituitary adenomas in which the $\mathrm{Ki}-67$ ranged from 0.1 to $2.8 \%$, with higher values in adenomas with infiltration of the dura mater. Two other studies $(26,28)$ carried out on samples of 31 and 70 pituitary adenomas, respectively, also associated higher Ki-67 indexes with tumor invasion degree. The same results were confirmed later $(31,33)$. However, in two other studies $(12,28)$, an overlap of Ki-67 index values was found in noninvasive adenomas, invasive adenomas, and pituitary carcinomas. In the present study, an overlap of MIB-1 values was also found between invasive and noninvasive adenomas. Thus, low values do not necessarily indicate the non-aggressive evolution of the tumor.

In the series of studies evaluating cell proliferation on the basis of the MIB-1 antibody in pituitary adenomas, an effort has been made to define an index value that could serve as a cut-off to distinguish between invasive adenomas of benign behavior and those of aggressive behavior. However, there is no consensus in the literature about this value. In a particular series (28), an index of $3 \%$ was successful in distinguishing between invasive and noninvasive adenomas with a specificity of $97 \%$ and a sensitivity of $73 \%$. Later, a cut-off value of 
$1 \%$ was proposed (31). Mastronardi et al. (12) established two different thresholds for the Ki-67 index: one for invasive adenomas $(3.5 \%)$ and the other for adenomas with invasion of the cavernous sinus (5\%). In their series, these investigators proposed a scaling system for invasion of pituitary adenomas according to the mean $\mathrm{Ki}-67$ index: grade zero, noninvasive adenomas with MIB1 up to $2.01 \%$; grade 1 , invasive adenomas with MIB-1 between 2.01 to 3.71\%; grade 2, adenomas with infiltration of the cavernous sinus and MIB-1 between 3.71 and 5.58\%, and grade 3 , pituitary carcinomas with a Ki67 index of $36 \%$ (one case). In the series described here, it was not possible to establish a value distinguishing invasive from noninvasive adenomas because of the considerable overlap of MIB-1 values between these two groups, although the results allow us to conclude that tumoral proliferative activity as evaluated on the basis of the MIB-1 antibody was significantly higher in invasive adenomas. Although low Ki-67 indexes do not exclude the possibility of tumor regrowth, the establishment of cut-off points associated with aggressive tumor behavior could be used in the future as an aid for therapeutic decision making.

\section{References}

1. Elster AD (1993). Modern imaging of the pituitary. Radiology, 187: 1-14.

2. Burrow GN, Wortzman G, Rewcastle NB, Holgate RC \& Kovacs K (1981). Microadenomas of the pituitary and abnormal sellar tomograms in an unselected autopsy series. New England Journal of Medicine, 304: 156-158.

3. Asa SL (1999). Pathology of pituitary tumors. Endocrinology and Metabolism Clinics of North America, 28: 13-43.

4. Shibuya M, Saito F, Miwa $T$, Davis RL, Wilson CB \& Hoshino $T$ (1992). Histochemical study of pituitary adenomas with Ki-67 and anti-DNA polymerase $\alpha$ monoclonal antibodies, bromodeoxyuridine labeling, and nucleolar organizer region counts. Acta Neuropathologica, 84: 178-183.

5. Gerdes J, Schwab U, Lemke H \& Stein H (1983). Production of a mouse monoclonal antibody reactive with a human nuclear antigen associated with cell proliferation. International Journal of Cancer, 31: 13-20.

6. Barbosa-Coutinho LM, Antunes ACM, Azambuja NA, Geyer GR, Gross JL, Ferreira NP, Lopes NM, Reichel CL \& Zettler CG (1989). Adenomas da hipófise. Estudo imuno-histoquímico de 167 casos. Arquivos de Neuro-Psiquiatria, 47: 308-312.

7. Mindermann T \& Wilson CB (1994). Age-related and gender-related occurrence of pituitary adenomas. Clinical Endocrinology, 41: 359364.

8. Scheithauer BW, Kovacs KT, Laws Jr ER \& Randall RV (1986). Pathology of invasive pituitary tumors with special reference to functional classification. Journal of Neurosurgery, 65: 733-744.

9. Sautner D \& Saeger W (1991). Invasiveness of pituitary adenomas. Pathology, Research and Practice, 187: 632-636.

10. Ahmadi J, North CM, Segall HD, Zee CS \& Weiss MH (1985). Cavernous sinus invasion by pituitary adenomas. American Journal of Neuroradiology, 6: 893-898.

11. Kawamoto H, Uozumi T, Kawamoto K, Arita K, Yano T \& Hirohata T (1995). Analysis of the growth rate and cavernous sinus invasion of pituitary. Acta Neurochirurgica, 136: 37-43.

12. Mastronardi L, Guiducci A, Spera C, Puzzilli F, Liberati F \& Maira G
(1999). Ki-67 labelling index and invasiveness among anterior pituitary adenomas: analysis of 103 cases using the MIB-1 monoclonal antibody. Journal of Clinical Pathology, 52: 107-111.

13. Linden MD, Torres FX, Kubus J \& Zarbo RJ (1992). Clinical application of morphologic and immunocytochemical assessments of cell proliferation. American Journal of Clinical Pathology, 97 (Suppl): S4S13.

14. Landolt AM, Shibata T \& Kleihues P (1987). Growth rate of human pituitary adenomas. Journal of Neurosurgery, 67: 803-806.

15. Knosp E, Kitz K \& Perneczky A (1989). Proliferation activity in pituitary adenomas: measurement by monoclonal antibody Ki-67. Neurosurgery, 25: 927-930.

16. Kitz K, Knosp E, Koos WT \& Korn A (1991). Proliferation in pituitary adenomas: measurement by MAB KI-67. Acta Neurochirurgica, 53 (Suppl): 60-64.

17. Tsanaclis AM, Robert F, Michaud J \& Brem S (1991). The cycling pool of cells within human brain tumors: in situ cytokinetics using the monoclonal antibody Ki-67. Canadian Journal of Neurological Sciences, 18: 12-17.

18. Knosp E, Steiner E, Kitz K \& Matula C (1993). Pituitary adenomas with invasion of the cavernous sinus space: a magnetic resonance imaging classification compared with surgical findings. Neurosurgery, 33: 610-618.

19. Raghavan R, Harrison D, Ince PG, James RA, Daniels $M$, Birch $P$, Caldwell GI \& Kendall-Taylor P (1994). Oncoprotein immunoreactivity in human pituitary tumours. Clinical Endocrinology, 40: 117-126.

20. Krämer A, Saeger W, Tallen G \& Lüdecke DK (1994). DNA measurement, proliferation markers, and other factors in pituitary adenomas. Endocrine Pathology, 5: 198-211.

21. Gandour-Edwards R, Kapadia SB, Janecka IP, Martinez AJ \& Barnes $L$ (1995). Biologic markers of invasive pituitary adenomas involving the sphenoid sinus. Modern Pathology, 8: 160-164.

22. Lübke D, Saeger W \& Lüdecke DK (1995). Proliferation markers and EGF in ACTH-secreting adenomas and carcinomas of the pituitary. Endocrine Pathology, 6: 45-55.

23. Key G, Becker MH, Baron B, Duchrow M, Schlüter C, Flad HD \& 
Gerdes J (1993). New Ki-67-equivalent murine monoclonal antibodies (MIB 1-3) generated against bacterially expressed parts of the Ki-67 cDNA containing three 62 base pair repetitive elements encoding for the Ki-67 epitope. Laboratory Investigation, 68: 629639.

24. Kelleher L, Magee HM \& Dervan PA (1994). Evaluation of cellproliferation antibodies reactive in paraffin sections. Applied Immunohistochemistry, 2: 164-170.

25. Asano K, Kubo O \& Tajika Y (1996). The relationship between cell proliferation and secretory activity in pituitary tumors. A review of 63 cases. No To Shinkei, 48: 543-549.

26. Daita G \& Yonemasu Y (1996). Dural invasion and proliferative potential of pituitary adenomas. Neurologia Medico-Chirurgica, 36: 211-214.

27. Ekramullah SM, Saitoh Y \& Arita N (1996). The correlation of Ki-67 staining indices with tumour doubling times in regrowing non-functioning pituitary adenomas. Acta Neurochirurgica, 138: 1449-1455.

28. Thapar K, Kovacs K, Scheithauer BW, Stefaneanu L, Horvath E, Pernicone PJ, Murray D \& Laws Jr ER (1996). Proliferative activity and invasiveness among pituitary adenomas and carcinomas: an analysis using the MIB-1 antibody. Neurosurgery, 38: 99-107.

29. Atkin SL, Green VL, Hipkin LJ, Landolt AM, Foy PM, Jeffreys RV \& White MC (1997). A comparison of proliferation indices in human anterior pituitary adenomas using formalin-fixed tissue and in vitro cell culture. Journal of Neurosurgery, 87: 85-88.

30. Delgrange E, Trouillas J, Maiter D, Donckier J \& Tourniaire J (1997). Sex-related difference in the growth of prolactinomas: a clinical and proliferation marker study. Journal of Clinical Endocrinology and Metabolism, 82: 2101-2107.

31. Mizoue $T$, Kawamoto $H$, Arita K, Kurisu K, Tominaga A \& Uozumi $T$ (1997). MIB1 immunopositivity is associated with rapid regrowth of pituitary adenomas. Acta Neurochirurgica, 139: 426-432.

32. Bertholon-Gregoire M, Trouillas J, Guigard HP, Loras B \& Tourniaire $\mathrm{J}$ (1999). Mono- and plurihormonal thyrotropic pituitary adenomas: pathological, hormonal and clinical studies in 12 patients. European Journal of Endocrinology, 140: 519-527.

33. Zhao D, Tomono $Y$ \& Nose T (1999). Expression of P27kip1 and Ki-67 in pituitary adenomas: an investigation of markers of adenoma invasiveness. Acta Neurochirurgica, 141: 187-192.

34. Yonezawa K, Tamaki N \& Kokunai T (1997). Clinical features and growth fractions of pituitary adenomas. Surgical Neurology, 48: 494-500.

35. Jaffrain-Rea ML, Di Stefano D, Minniti G, Bultrini A, Ferretti E, Baldelli R, Tamburrano G, Gulino A \& Cantore G (1998). Ki-67 index as a marker of cell proliferation in pituitary tumors. Abstracts of IV European Congress of Endocrinology, Sevilla, Spain, May 9-13, 1998, P3-100. 